\title{
THE ADVECTION OF SUPERGRANULES BY THE SUN'S AXISYMMETRIC FLOWS
}

\author{
David H. Hathaway ${ }^{1}$, Peter E. Williams ${ }^{2}$, Kevin Dela Rosa ${ }^{3}$, and Manfred Cuntz ${ }^{4}$ \\ ${ }^{1}$ NASA Marshall Space Flight Center, Huntsville, AL 35812, USA; david.hathaway@ nasa.gov \\ 2 NASA Goddard Space Flight Center, Greenbelt, MD 20771, USA; peter.williams@nasa.gov \\ ${ }^{3}$ School of Computer Science, Carnegie Mellon University, Pittsburgh, PA 15213, USA \\ ${ }^{4}$ Department of Physics, University of Texas at Arlington, Arlington, TX 76019, USA; cuntz@uta.edu \\ Received 2010 August 25; accepted 2010 October 6; published 2010 November 23
}

\begin{abstract}
We show that the motions of supergranules are consistent with a model in which they are simply advected by the axisymmetric flows in the Sun's surface shear layer. We produce a 10 day series of simulated Doppler images at a 15 minute cadence that reproduces most spatial and temporal characteristics seen in the $S O H O /$ MDI Doppler data. Our simulated data have a spectrum of cellular flows with just two components-a granule component that peaks at spherical wavenumbers of about 4000 and a supergranule component that peaks at wavenumbers of about 110 . We include the advection of these cellular components by the axisymmetric flows-differential rotation and meridional flow-whose variations with latitude and depth (wavenumber) are consistent with observations. We mimic the evolution of the cellular pattern by introducing random variations to the phases of the spectral components at rates that reproduce the levels of cross-correlation as functions of time and latitude. Our simulated data do not include any wave-like characteristics for the supergranules yet can reproduce the rotation characteristics previously attributed to wave-like behavior. We find rotation rates which appear faster than the actual rotation rates and attribute this to projection effects. We find that the measured meridional flow does accurately represent the actual flow and that the observations indicate poleward flow to $65^{\circ}-70^{\circ}$ latitude with equatorward countercells in the polar regions.
\end{abstract}

Key words: Sun: granulation - Sun: photosphere - Sun: rotation - Sun: surface magnetism

Online-only material: color figure

\section{INTRODUCTION}

Supergranules are cellular flow structures observed in the solar photosphere with typical diameters of about $30 \mathrm{Mm}$, lifetimes of about 1 day, and flow velocities of $300 \mathrm{~m} \mathrm{~s}^{-1}$ (Rieutord \& Rincon 2010). They cover the entire surface of the Sun except for the immediate surroundings of sunspots.

Supergranules were discovered by Hart (1954) but it was Leighton et al. (1962) who suggested that these cellular flows were convective structures and coined the term "supergranule." However, Worden (1975), and others since, had difficulty detecting any associated thermal features (i.e., hot cell centers) consistent with a thermal convection origin. Recently, however, Meunier et al. (2007) have reported the detection of a small temperature excess at cell centers of about $0.8-2.8 \mathrm{~K}$.

Supergranules are intimately involved with the structure and evolution of the magnetic field in the photosphere. Simon \& Leighton (1964) found that the magnetic structures of the chromospheric network are located at the boundaries of these cells. Leighton (1964) quickly suggested that the random walk of magnetic elements by the evolving supergranules could transport following polarity elements poleward to reverse the Sun's polar field each solar cycle while leading polarity elements would be transported across the equator where they would meet and cancel their opposite polarity counterparts. While we now recognize that Leighton over estimated the effective diffusivity implied by this random walk (and that the Sun's poleward meridional flow provides the missing transport), supergranule diffusion is still not fully constrained or understood. Leighton's initial estimate of $770-1540 \mathrm{~km}^{2} \mathrm{~s}^{-1}$ for the diffusivity was reduced to $200-400 \mathrm{~km}^{2} \mathrm{~s}^{-1}$ by Mosher (1977), who studied the displacements of chromospheric network elements. Schrijver \& Martin (1990) measured the displacements of the magnetic elements themselves using magnetograms and found a value of $250 \mathrm{~km}^{2} \mathrm{~s}^{-1}$ in plage surrounding active regions but only $110 \mathrm{~km}^{2} \mathrm{~s}^{-1}$ in the active regions themselves. Models for the surface magnetic flux transport typically use values about twice this size - van Ballegooijen et al. (1998) used $450 \mathrm{~km}^{2} \mathrm{~s}^{-1}$ while Wang et al. (2002) used $500 \mathrm{~km}^{2} \mathrm{~s}^{-1}$. Simon et al. (1995) used a relatively simple kinematic model of supergranule diffusion and arrived at a similar estimate of $500-700 \mathrm{~km}^{2} \mathrm{~s}^{-1}$.

Supergranules are embedded in a surface shear layer of their own making. Foukal \& Jokipii (1975) and Foukal (1979) suggested that the radial flows within the supergranules would conserve angular momentum and produce a shear layer with slower rotation at the surface and more rapid rotation below-consistent with the earlier observations of Howard \& Harvey (1970) that the sunspots rotate about 5\% faster than the surrounding surface plasma. The production of this shear layer was successfully modeled in spherical shells by Gilman \& Foukal (1979) and in plane-parallel layers by Hathaway (1982). The theoretical arguments suggest a change in rotation frequency such that $\delta \Omega \sim-2 \Omega \delta r / r$. In addition, Hathaway (1982) found that a meridional flow was also produced with poleward flow near the surface and equatorward flow at the bottom.

The surface shear layer has been probed with helioseismic techniques which confirm the existence of the layer but give somewhat different results for the detailed structure in depth and latitude. Thompson et al. (1996) used the global oscillation modes from the Global Oscillation Network Group (GONG) instruments and found that the rotation rate increased inward to a depth of about $35 \mathrm{Mm}(\delta r / r \sim 5 \%)$ and that the rate of increase was larger at the equator than at $30^{\circ}$ latitude. Schou et al. (1998) found similar results from global modes in the Solar and Heliospheric Observatory ( $\mathrm{SOHO}$ ) Michelson Doppler Imager (MDI) instrument data but noted that the shear 
appeared to disappear by $60^{\circ}$ latitude and perhaps reverse sign at higher latitudes. Corbard \& Thompson (2002) studied the radial gradient in the near surface rotation (depths of 3-8 Mm) in some detail using analysis of the $f$-mode frequency splittings from MDI data and found a linear increase with depth at the theoretical rate for latitudes below $30^{\circ}$ but also found that this gradient dropped below its theoretical value and reversed sign at $\sim 60^{\circ}$.

Basu et al. (1999) used the local helioseismology technique of ring-diagram analysis. They found a thin $(4 \mathrm{Mm})$ outer shear layer in which the shear did not appear to reverse at high latitudes and a deeper shear layer in which it did. They also found a poleward meridional flow which increased in amplitude across the thin outer shear layer but then remained nearly constant across the inner shear layer. Their meridional flow had peak velocities of about $50 \mathrm{~m} \mathrm{~s}^{-1}$ at $50^{\circ}$ latitude. Giles et al. (1997) used a time-distance local helioseismology technique and found a meridional flow with a peak velocity of only about $24 \mathrm{~m} \mathrm{~s}^{-1}$ at $45^{\circ}$ latitude and indicated that it was constant to a depth of about $26 \mathrm{Mm}$.

The observed rotation of the supergranules has added further mystery to their nature. (The mechanism that selects the characteristic size of supergranules is perhaps the biggest mystery.) Duvall (1980) cross-correlated the equatorial Doppler velocity patterns and found that the supergranules rotated more rapidly than the plasma at the photosphere and that even faster rotation rates were obtained when there were longer $(24 \mathrm{hr}$ versus $8 \mathrm{hr}$ ) time intervals between Doppler images. He attributed this behavior to the surface shear layer in which larger, longer-lived, cells extend deeper into the more rapidly rotating layers. Snodgrass \& Ulrich (1990) used data from Mount Wilson Observatory to find the rotation rate at different latitudes and noted that the rotation rates for the Doppler pattern were some $4 \%$ faster than the spectroscopic rate and, mysteriously, some $2 \%$ faster than the small magnetic features that are observed to outline their borders. More recently, Beck \& Schou (2000) used a Fourier transform method to find that the larger features do rotate more rapidly than the smaller features and that the low wavenumber components of the Doppler pattern rotate more rapidly than the plasma at any level within the surface shear layer. This led them to suggest that supergranules have wave-like characteristics with a preference for prograde propagation.

Hathaway et al. (2006) showed that this "super-rotation" of the Doppler pattern could be attributed to projection effects associated with the Doppler signal itself. As the velocity pattern rotates across the field of view the observed line-of-sight component is modulated in a way that essentially adds another half-wave to the pattern and gives a higher rotation rate that increases proportionally with decreasing wavenumber. They took a fixed velocity pattern (which had spatial characteristics that matched the SOHO/MDI data) and rotated it rigidly to show this "super-rotation" effect. While this indicated that the Doppler projection effect should be accounted for, the fixed pattern could not account for all the variations reported by Beck \& Schou (2000).

Gizon et al. (2003) used time-distance helioseismology to find the supergranular flow (rather than direct Doppler measurements) and Schou (2003) "divided-out" the line-ofsight modulation. Both studies found slower rotation rates that matched that of the magnetic features but saw evidence for wavelike prograde and retrograde moving components. Meunier \& Roudier (2007) compared rotation rates obtained by tracking
Doppler features, magnetic features, and divergence features (which were, in turn, derived from correlation tracking of smaller intensity features). While they concluded that projection effects do influence the rotation rate determined from the Doppler features, they found that the magnetic features rotate more slowly than the supergranules.

In this paper, we report on our analyses of data from the SOHO/MDI instrument (Scherrer et al. 1995) and from simulated data in which the supergranules are simply advected by differential rotation and meridional flow that vary with latitude and depth. The simulated data are designed to faithfully mimic the $S O H O / M D I$ data (the same data that were analyzed in Beck \& Schou 2000 and Schou 2003) with simple assumptions about the dynamical structure of the surface shear layer. The analyses include reproductions of those done in earlier studies. Through the simulations we can better determine the actual differential rotation and meridional flow profiles consistent with the Doppler observations (which are subject to line-of-sight projection effects).

\section{THE DATA}

The full-disk Doppler images from $\mathrm{SOHO/MDI}$ are obtained at a 1 minute cadence to resolve the temporal variations associated with the $p$-mode oscillations. We (cf. Hathaway et al. 2000 and Beck \& Schou 2000) have temporally filtered the images to remove the $p$-mode signal by using a 31 minute long tapered Gaussian with a FWHM of 16 minutes on sets of 31 images that were de-rotated to register each to the central image. These filtered images were formed at 15 minute intervals over the 60 day MDI Dynamics Run in 1996. This filtering process effectively removes the $p$-mode signal and leaves behind the Doppler signal from flows with temporal variations longer than about 16 minutes.

Supergranules, with typical wavenumbers of about 110 , are very well resolved in these data (at disk center wavenumbers up to 1500 are resolved). While granules are not well resolved, they do appear in the data as pixel-to-pixel and image-toimage "noise," as a convective blueshift (due to the correlation between brightness and updrafts), and as resolved structures for the largest members. These data are prepared for studying the cellular features by first measuring and removing the Doppler signals due to (1) observer motion, (2) convective blueshift, (3) differential rotation, and (4) the axisymmetric meridional flow. The data are then mapped onto heliographic coordinates with equal spacing in both longitude and latitude. This mapping includes accounting for the position angle and tilt angle of the Sun's rotation axis as well as the misorientation of the SOHO/MDI detector. Note that the position angle and tilt angle of the Sun's rotation axis determined by Carrington (1863) have been found to be in error by about 0.1 by Beck \& Giles (2005) and Hathaway \& Rightmire (2010). This correction is included in this study.

Korzennik et al. (2004) have found that the MDI image may be slightly elliptical (a 0.5 out of 490 pixel difference) and should have a cubic distortion in a radius of about $0.1 \%$. While they give the orientation of the semimajor axis of the ellipse as $56^{\circ}$ counterclockwise from the detector's "vertical" axis, the online MDI documentation gives the angle as $75^{\circ}$ counterclockwise. Given the uncertainty in this elliptical distortion, we have not included it in our mapping. We have, however, included a 0.5 pixel offset (image origin at bottom left corner of bottom left pixel instead of the center of that pixel as indicated in the MDI documentation) which appeared to give cleaner results over time 


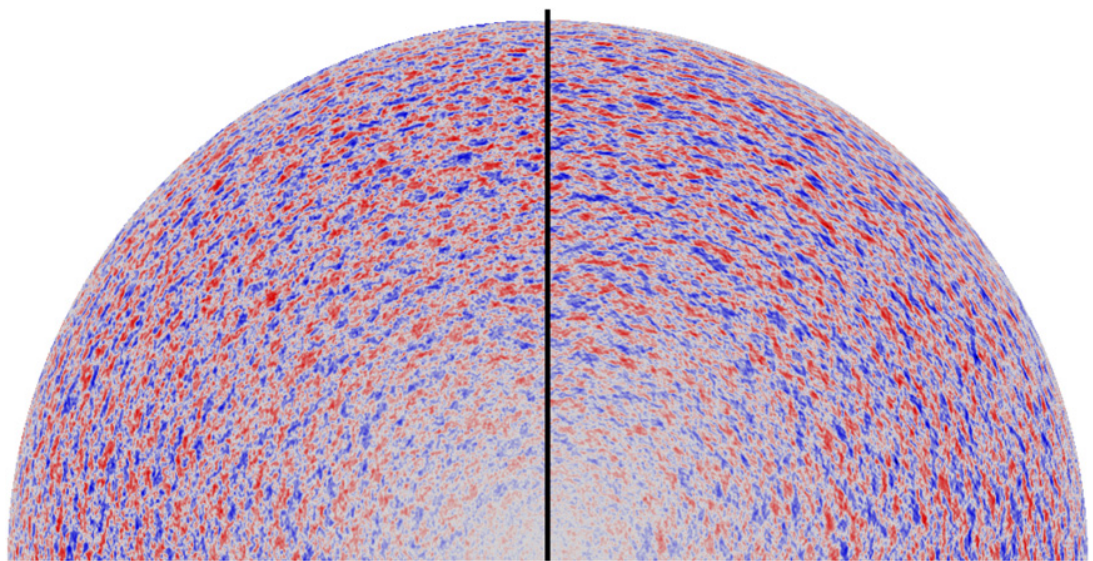

Figure 1. Doppler velocity images (MDI on left, simulation on right) in which the observer's motion, the convective blueshift, and the Doppler signals due to the axisymmetric flows - differential rotation and meridional circulation—are removed to better reveal the cellular structures (supergranules). The spatial characteristics are visually indistinguishable.

(A color version of this figure is available in the online journal.)

in the analysis of Hathaway \& Rightmire (2010). The elliptical distortion could introduce small $(0.1 \%)$ systematic changes to the differential rotation and meridional flow profiles we find in the mapped MDI data.

The simulated data are constructed in the manner described previously (Hathaway 1988, 1992; Hathaway et al. 2000, 2002) from vector velocities with

$$
\begin{aligned}
& V_{r}(\theta, \phi)=\sum_{l=0}^{l \max } \sum_{m=-l}^{l} R_{l}^{m} Y_{l}^{m}(\theta, \phi) \\
& V_{\theta}(\theta, \phi)=\sum_{l=1}^{l \max } \sum_{m=-l}^{l} S_{l}^{m} \frac{\partial Y_{l}^{m}(\theta, \phi)}{\partial \theta} \\
& +\sum_{l=1}^{l \max } \sum_{m=-l}^{l} T_{l}^{m} \frac{1}{\sin \theta} \frac{\partial Y_{l}^{m}(\theta, \phi)}{\partial \phi} \\
& V_{\phi}(\theta, \phi)=\sum_{l=1}^{l \max } \sum_{m=-l}^{l} S_{l}^{m} \frac{1}{\sin \theta} \frac{\partial Y_{l}^{m}(\theta, \phi)}{\partial \phi} \\
& -\sum_{l=1}^{l \max } \sum_{m=-l}^{l} T_{l}^{m} \frac{\partial Y_{l}^{m}(\theta, \phi)}{\partial \theta},
\end{aligned}
$$

where $Y_{\ell}^{m}(\theta, \phi)$ is the spherical harmonic function of degree $\ell$ and azimuthal order $m, \theta$ is the colatitude measured southward from the north pole, and $\phi$ is the longitude measured prograde from the central meridian or some fixed longitude. The complex quantities $R_{l}^{m}, S_{l}^{m}$, and $T_{l}^{m}$ are the spectral coefficients for the radial, poloidal, and toroidal components, respectively.

To simulate the observed line-of-sight velocity, the spectral coefficients from $\ell=0$ to $\ell=1500$ are specified and the three vector velocity components are calculated using Equations (1)-(3) on a grid with 1500 points in latitude and 4096 points in longitude. The Doppler velocity image is constructed by determining the longitude and latitude at a point on the image, finding the vector velocity at that point using bi-cubic interpolation, and then projecting that vector velocity onto the line of sight using

$$
\begin{aligned}
& V_{\mathrm{los}}(\theta, \phi)=V_{r}(\theta, \phi) \sin B_{0} \cos \theta \\
& \quad+V_{r}(\theta, \phi) \cos B_{0} \sin \theta \cos \phi+V_{\theta}(\theta, \phi) \sin B_{0} \sin \theta \\
& \quad-V_{\theta}(\theta, \phi) \cos B_{0} \cos \theta \cos \phi+V_{\phi}(\theta, \phi) \cos B_{0} \sin \phi,
\end{aligned}
$$

where $B_{0}$ is the latitude at disk center (or equivalently the tilt of the Sun's north pole toward the observer) and velocities away from the observer are taken to be positive. The line-of-sight velocity at an array of 49 points within each pixel is determined and an average is taken to simulate the integration over a pixel with the MDI instrument.

With the current simulations the instrumental blurring is treated in a more realistic manner. Previously the Doppler velocity image itself was convolved with a point-spread function approximating that found for MDI by Korzennik et al. (2004). Here, we make red and blue intensity images from our Doppler velocity image and a simple limb darkened intensity image, convolve those with an MDI point-spread function, and construct the blurred Doppler velocity image from the difference divided by the sum.

This process yields Doppler velocity images that are visually indistinguishable from MDI Doppler velocity images. A velocity image comparison is shown in Figure 1.

The velocity pattern is evolved in time by introducing changes to the spectral coefficients based on two processes- the advection by the axisymmetric flows (differential rotation and meridional flow) and random processes that lead to the finite lifetimes of the cells.

The advection is governed by an advection equation

$$
\frac{\partial w}{\partial t}=-\frac{V(\theta)}{r} \frac{\partial w}{\partial \theta}-\frac{U(\theta)}{r \sin \theta} \frac{\partial w}{\partial \phi},
$$

where $w$ is a velocity component, $U(\theta)=r \sin \theta \Omega(\theta)$ gives the differential rotation profile, and $V(\theta)$ gives the meridional flow velocity profile. Representing $w$ as a series of spherical harmonic components (Equations (1)-(3)) and projecting this advection equation onto a single spherical harmonic gives a series of coupled equations for the evolution of the spectral coefficients (see the Appendix). Solid body rotation simply introduces a constantly increasing phase for each coefficient. Differential rotation couples the phase change in one spectral coefficient to spectral coefficients with wavenumbers $\ell \pm 2$ and $\ell \pm 4$ for differential rotation of the form

$$
\Omega(\theta)=\Omega_{0}+\Omega_{2} \cos ^{2} \theta+\Omega_{4} \cos ^{4} \theta,
$$

while a simple but reasonable meridional flow profile with

$$
V(\theta)=V_{0} \cos \theta \sin \theta
$$


couples one spectral coefficient to spectral coefficients with wavenumbers $\ell \pm 2$. (Spherical harmonics have fixed latitudinal structure. Spectral power must pass from one spherical harmonic component to another in order to move a feature in latitude.)

These cellular flows are embedded in the Sun's surface shear layer. We approximate the change in the rotation rate in the outermost $5 \%$ of the Sun as reported by Corbard \& Thompson (2002) and Howe et al. (2007) with

$$
\Omega(r, \theta)=\Omega(\theta) f(r),
$$

where

$$
f(r)=1+0.038\left[1-e^{-55\left(1-r / R_{\odot}\right)}\right]
$$

and the latitude dependence is given by

$$
\begin{aligned}
& \frac{\Omega_{0}}{2 \pi}=452 \mathrm{nHz}\left(14.07 \mathrm{day}^{-1}\right) \\
& \frac{\Omega_{2}}{2 \pi}=-55 \mathrm{nHz}\left(-1.75 \mathrm{day}^{-1}\right) \\
& \frac{\Omega_{4}}{2 \pi}=-75 \mathrm{nHz}\left(-2.30 \mathrm{day}^{-1}\right) .
\end{aligned}
$$

Assuming that the cells extend to depths similar to their horizontal dimensions, and that they are advected at flow rates representative of that depth, Equation (9) is transformed into a function of $\ell$ with

$$
f(\ell)=1+0.038\left[1-e^{-90 \pi / \ell}\right] .
$$

This shear layer profile is illustrated in Figure 2 along with the gradients expected from theoretical arguments for flows that conserve angular momentum. We assume a meridional flow which is constant with depth across this layer and has a latitude dependence characterized by

$$
V_{0}=-30 \mathrm{~m} \mathrm{~s}^{-1}
$$

which gives a peak meridional flow velocity of $15 \mathrm{~m} \mathrm{~s}^{-1}$ at $45^{\circ}$ latitude.

The finite lifetimes for the cells are simulated by introducing random perturbations to the spectral coefficient phases. The size of these perturbations increases with wavenumber to give shorter lifetimes to smaller cells with

$$
\delta \Phi_{\ell}^{m} \propto \sqrt{\Delta t / \tau(\ell)}
$$

where $\delta \Phi_{\ell}^{m}$ is the change in phase for a complex spectral coefficient of degree $\ell$ and order $m, \Delta t$ is the time interval between simulated Doppler images, and $\tau(\ell)$ is proportional to the lifetime for a spectral component of degree $\ell$. Lifetimes are well approximated by a turnover time for turbulent convective flows. The cellular flow velocities are roughly proportional to $\ell$ while their diameters are inversely proportional to $\ell$. The turnover times should then be inversely proportional to $\ell^{2}$. We find a reasonable fit to the data using

$$
\tau(\ell)=6.5 \frac{100^{2}}{\ell^{2}} \mathrm{hr} .
$$
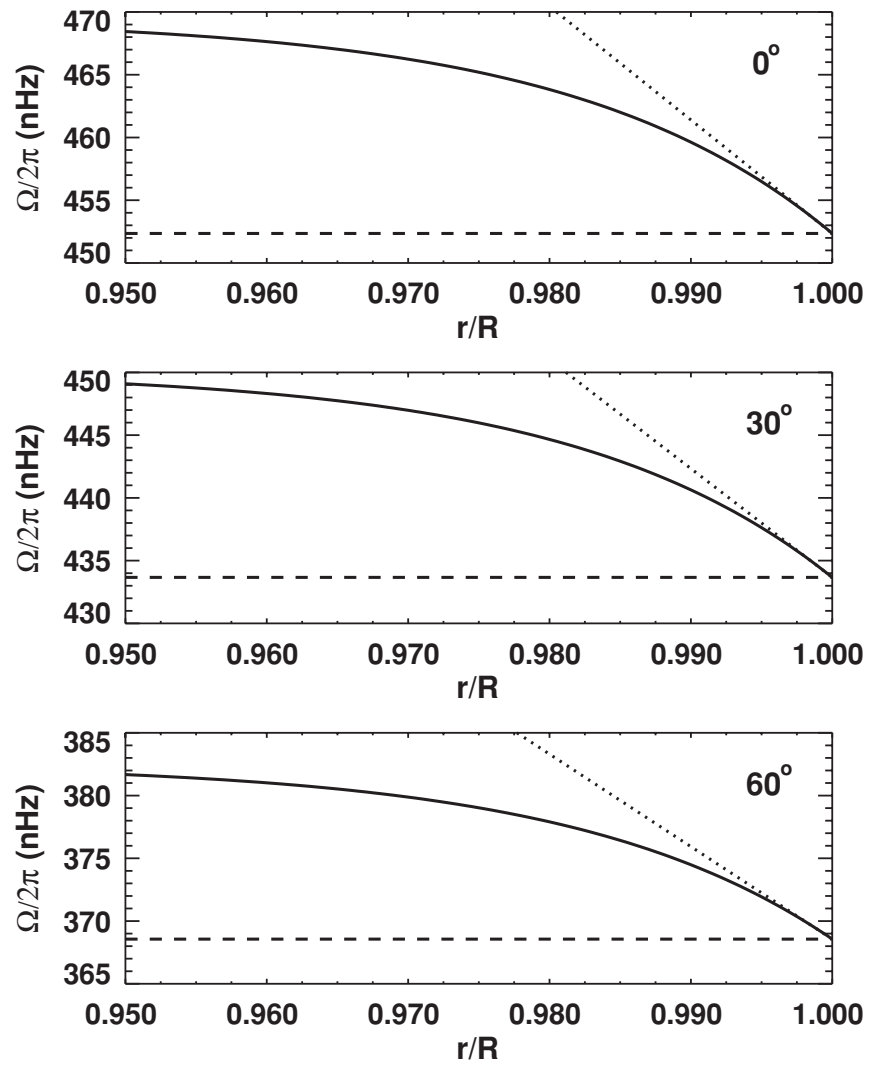

Figure 2. Assumed rotation rate as a function of radius in the surface shear layer for three different latitudes. The surface rotation rate at each latitude is indicated by the dashed lines. The variations in rotation rate for flows that conserve angular momentum from the surface inward are indicated by the dotted lines.

\section{THE ANALYSES}

Several analysis programs were applied to both the MDI data and the simulated data. Power spectra were obtained to characterize and compare the distribution of cell sizes and flow velocities. The rotation of the Doppler pattern was determined using multiple techniques based on previous studies. The meridional flow was measured based on the movement of the Doppler pattern and cell lifetimes were estimated from crosscorrelation analyses.

\subsection{Convection Spectra}

Convection spectra for individual images were obtained using the methods described by Hathaway (1987) and Hathaway (1992) — the Doppler signal due to the motion of the observer is removed, the convective blueshift signal is identified and removed, the data are mapped to heliographic coordinates, the axisymmetric flow signals due to differential rotation and meridional circulation are identified and removed, and the remaining signal is projected onto spherical harmonics. The averaged spectra from the 60 day 1996 MDI Dynamics Run and from our 10 day simulated data run are shown in Figure 3.

The match between these spectra is obtained by adjusting the input spectrum for the simulated data. This spectrum contains two Lorentzian-like spectral components-a supergranule component centered on $\ell \sim 110$ with a width of about 100 and a granule component centered on $\ell \sim 4000$ with a width of about 3000. The MDI spectrum is well matched with just these two components without the addition of the mesogranule com- 


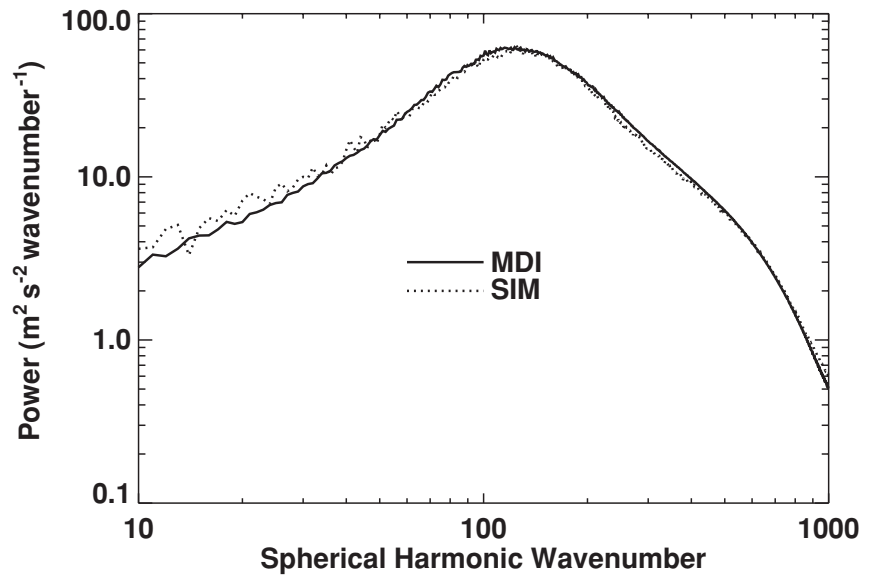

Figure 3. Power spectra from spherical harmonic analyses of MDI data (solid line) and simulated data (dotted line). The spectral match indicates that both data sets have very similar spatial structures. (The rapid drop in power for wavenumbers beyond 600 is largely due to foreshortening and instrumental blurring.)

ponent suggested by November et al. (1981). In fact, we have a distinct dip in the input spectrum at wavenumbers $\ell \sim 500$ that should be representative of mesogranules. Hathaway et al. (2000) showed that this dip is easily seen in the observed spectra from the MDI high-resolution data.

\subsection{Rotation}

Rotation information is obtained when additional analyses are applied to the data after they have been mapped onto heliographic coordinates. Longitudinal strips of these data, centered on latitudes from $75^{\circ}$ south to $75^{\circ}$ north, were crosscorrelated with corresponding strips from later images as was done by Duvall (1980) and Snodgrass \& Ulrich (1990). The shift in the location of the cross-correlation peak divided by the time difference gives the rotation rate.

Figure 4 shows the differential rotation from the crosscorrelation analysis. The profiles from the simulated data match those from the MDI data at all but the highest latitudes. Both data sets show faster rotation rates for longer time lags as noted by Duvall (1980) and Snodgrass \& Ulrich (1990). The match between the MDI data and the simulated data indicates that the latitudinal differential rotation profile is fairly well represented by Equation (6) with the coefficients given by Equations (10)-(12) derived from the helioseismic and direct Doppler studies. The differences at high latitude may be an indication that the shear layer does indeed disappear above about $50^{\circ}$ as reported by Schou et al. (1998) and Corbard \& Thompson (2002). This would give slower (more negative) differential rotation rates for the supergranules than used in the simulation with the shear layer shown in Figure 2. The MDI data also show slightly faster equatorial rotation. This suggests that either Equation (13) should be modified to give faster rotation at the lower wavenumbers or Equation (16) should be modified to give shorter lifetimes at the higher wavenumbers.

We reproduced the analysis of Beck \& Schou (2000). Lines of data from the heliographic maps were Fourier analyzed in longitude and those spectral coefficients were then Fourier analyzed in time over 10 day intervals. The power spectra were averaged over all latitude lines between $\pm 9^{\circ}$. The rotation rate for each wavenumber $\ell$ was determined by finding the temporal frequency of the center of gravity of the power using a frequency window of $\approx 58 \mu \mathrm{Hz}$ (and iterating on the position of that window four times) and then dividing the temporal frequency by the wavenumber.

Figure 5 shows the equatorial rotation rates as functions of wavenumber for both the MDI data and the simulated data. Here again we find a good match for all but the lowest wavenumbers. This indicates that Equation (13) gives a good wavenumber dependence for the rotation rate. The drop in rotation rate for the MDI data at wavenumbers below 30 is due to imaging artifacts that introduce signal at low temporal frequencies which can overlap with the rotation signal at low spatial frequencies. The effects of the line-of-sight projection on measured rotation rates, as discussed by Hathaway et al. (2006), are evident in the increase in the observed rotation rates above the input rates from Equation (13) for wavenumbers below $~ 100$. Although the higher noise level from the single 10 day simulation makes precise comparisons difficult, it does appear that the MDI data
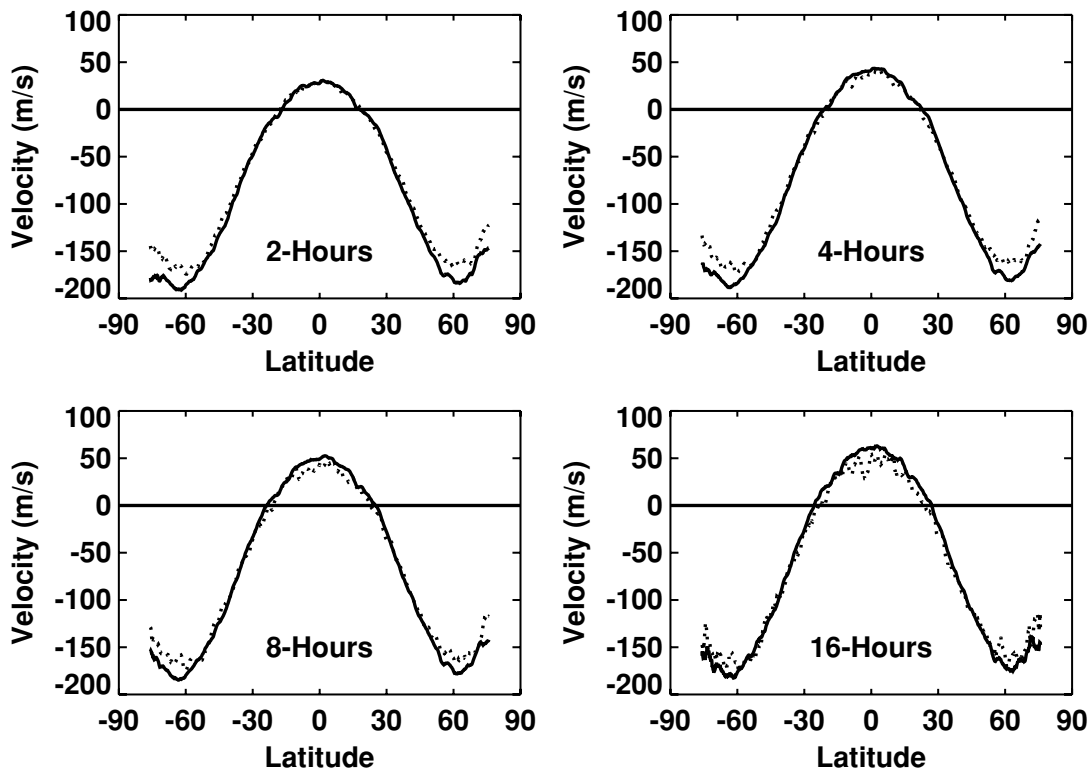

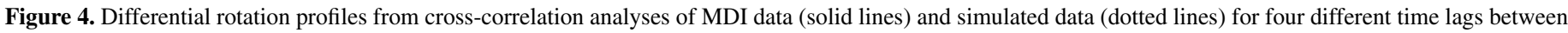
Doppler images $-2,4,8$, and $16 \mathrm{hr}$. All profiles are well matched except at the highest latitudes and near the equator for the longest time lags. 


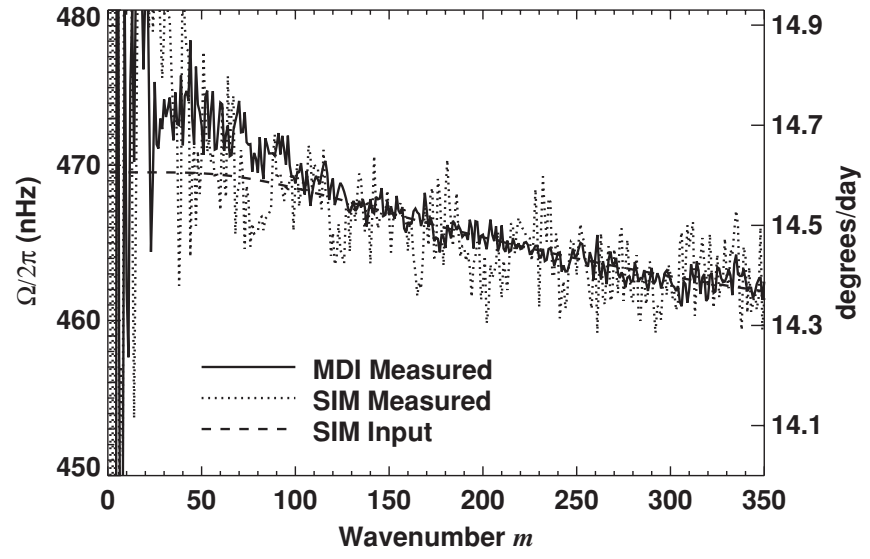

Figure 5. Equatorial rotation rates as functions of wavenumber from analyses of six 10 day sets of MDI data (solid line) and a single 10 day set of simulated data (dotted line). The equatorial rotation rates used as input (Equation (13)) are shown with the dashed line. The rotation rates match very well at all but the lowest wavenumbers. Rotation rates faster than the input rates at wavenumbers below about 100 are due to projection effects.

might be better fitted if the rotation rates given by Equation (13) were somewhat higher at wavenumbers below $\sim 100$. This would also produce a better fit for the differential rotation rates near the equator at the longer time lags.

We have also reproduced one of the analyses of Schou (2003). Lines of data from the heliographic maps are apodized and then multiplied by longitude-dependent weighting functions designed to remove the Doppler projection effect and to isolate either longitudinal motions or latitudinal motions. The weighting used to isolate longitudinal flows is based on the final term in Equation (4) but with $B_{0}=0$ and a constant of 0.01 added to $\sin \phi$ to avoid division by zero at the central meridian. The lines are shifted in longitude according to the differential rotation rate and then Fourier analyzed in space and time to obtain " $k-\omega$ " diagrams. Schou (2003) noted that these diagrams show power for both prograde $(\omega / k<0)$ and retrograde $(\omega / k>0)$ motion but with excess power in the prograde components.

Figure 6 shows the $k-\omega$ diagrams from 10 days of the MDI data and from the simulated data for latitudes between \pm 4.5 . Both show prograde and retrograde components as similarly shaped wedges of enhanced power extending from the origin to both positive and negative longitudinal wavenumbers. However, the MDI data do show excess power for prograde motion (power

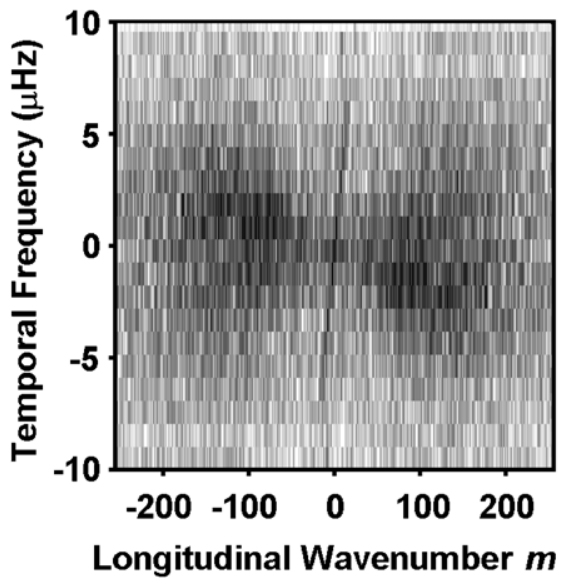

excess extending from the upper left to the lower right) that is not evident in the simulated data. (Note that running this analysis on the full 60 day run of MDI data shows a clear separation between prograde and retrograde motions.) This discrepancy may be produced by the advection of the supergranules by larger, nonaxisymmetric flows. This is discussed further in our conclusions (Section 4).

\subsection{Meridional Flow}

The meridional motion of the Doppler pattern can be measured by the cross-correlation method used to find the differential rotation profiles illustrated in Figure 4 or by using the apodizing/weighting method described by Schou (2003). Figure 7 shows a comparison of the meridional flow profiles obtained from the MDI data and the simulated data when we take $V_{0}=-30 \mathrm{~m} \mathrm{~s}^{-1}$, representing a poleward meridional flow with a peak velocity of $15 \mathrm{~m} \mathrm{~s}^{-1}$. The meridional flow we find for the Doppler features in the simulation very closely matches the input meridional flow (dashed lines in Figure 7). This indicates that the projection effects that produce the super-rotation of the Doppler features do not impact the measurements of the meridional flow.

It is clear that the actual meridional flow of the supergranules is more complicated than that used in our simulation. The meridional flow profiles for the supergranules shown in Figure 7 for the MDI data agree very well with those obtained by Gizon et al. (2003) and Schou (2003) using somewhat different methods. The MDI data indicate the presence of countercells in the polar regions during this 1996 data run that would be better represented by

$$
V(\theta)=-\left(50 \cos \theta-60 \cos ^{3} \theta\right) \sin \theta .
$$

In addition, the flow appears to weaken for longer time differences between the cross-correlated data. This suggests that the meridional flow in the surface shear layer decreases in amplitude with depth.

\subsection{Lifetimes}

The lifetimes of the cells can be estimated by comparing the strength of the cross-correlation coefficients for the Doppler features as functions of the time interval between Doppler images. Figure 8 shows the strength of the correlations as functions of latitude and time lag for both the MDI data

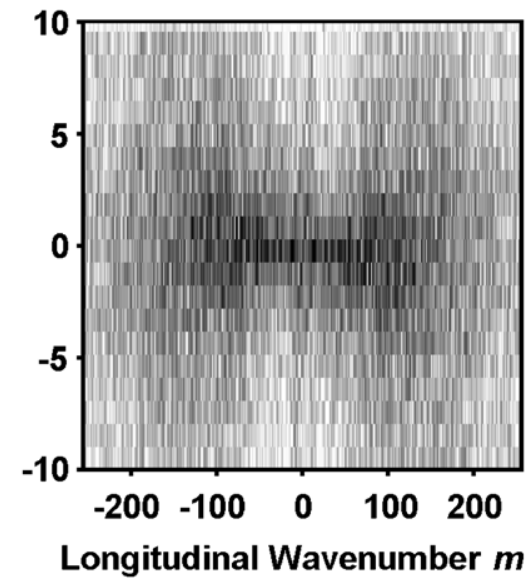

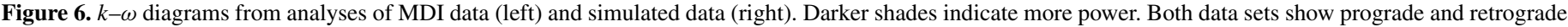

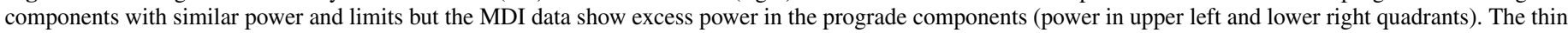
line from upper right to lower left in the MDI data is produced by imaging artifacts which appear to move retrograde at the Sun's rotation rate. 

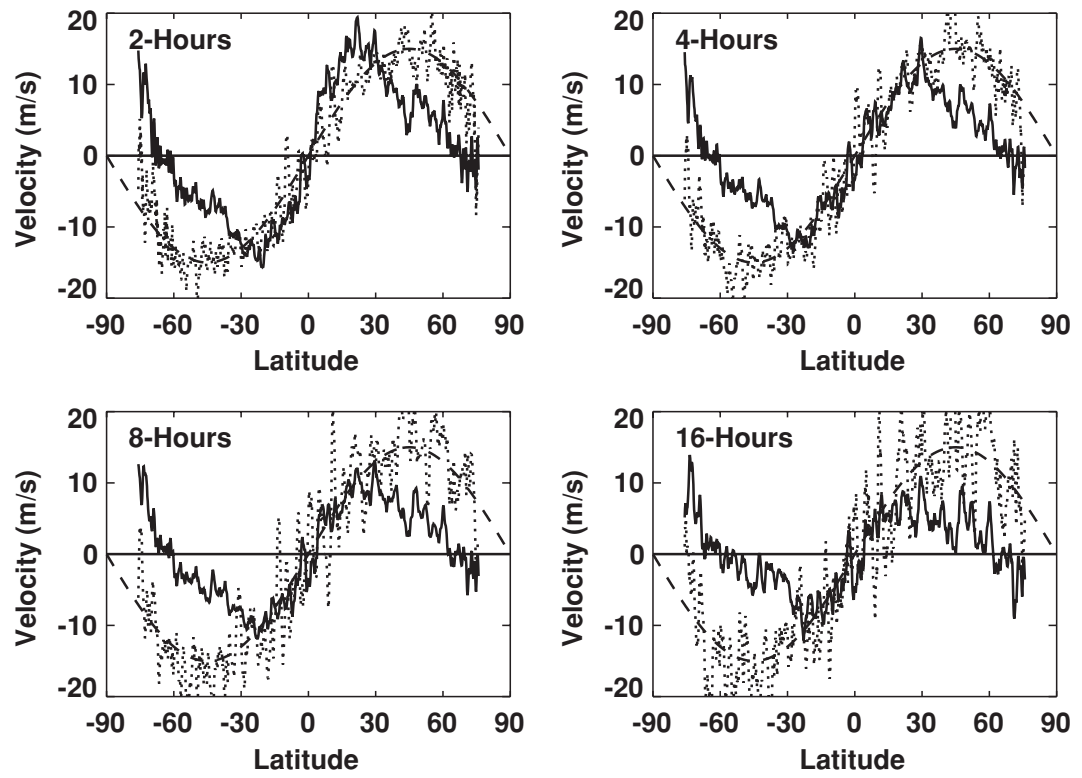

Figure 7. Meridional flow profiles from cross-correlation analyses of MDI data (solid lines) and simulated data (dotted lines) for four different time lags between Doppler images-2, 4, 8, and $16 \mathrm{hr}$. The meridional flow profile input to the simulation is shown by the dashed lines.

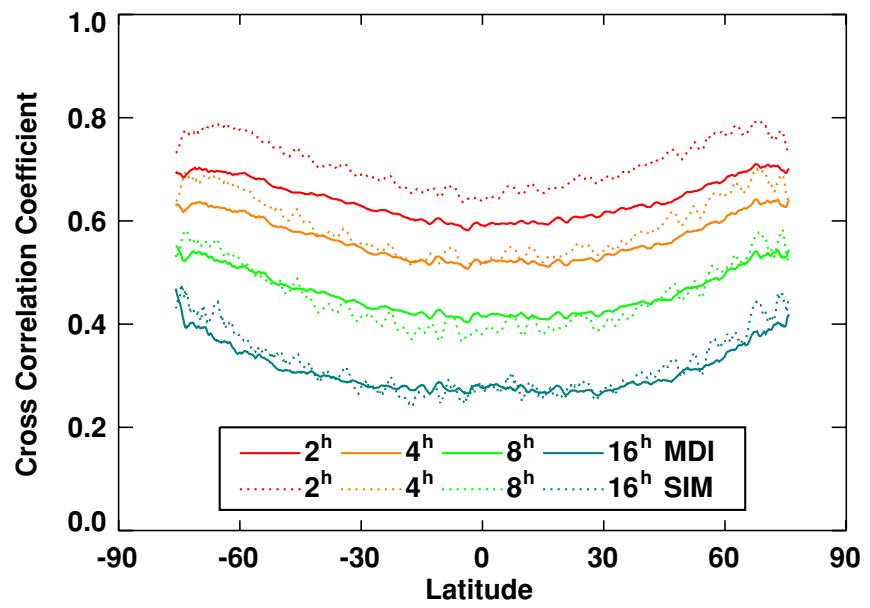

Figure 8. Cross-correlation peak profiles from analyses of MDI data (solid lines) and simulated data (dotted lines). The profiles are well matched at $\Delta t=4$ and $\Delta t=16 \mathrm{hr}$ but with slight differences at $\Delta t=2$ and $\Delta t=8 \mathrm{hr}$.

and the simulated data. The profiles are similar in shape but slightly flatter with the MDI data. The correlation coefficients are well matched at the equator for time differences of $4 \mathrm{hr}$ and $16 \mathrm{hr}$ but the simulation shows stronger correlations at $2 \mathrm{hr}$ and weaker correlations at $8 \mathrm{hr}$. This suggests that the formulae (Equations (15) and (16)) for the random phase changes to the spectral coefficients need slight modification. In particular, the cellular features that dominate at $\Delta t=2 \mathrm{hr}$ need shorter lifetimes.

\section{CONCLUSIONS}

We have produced simulated data in which the cellular structures (supergranules) are advected by differential rotation and meridional flow and evolved by uncorrelated random changes. When we compare results from analyses of these data with those from the MDI data we find that the simulated data exhibit many of the same characteristics as the MDI data-the visual structures, the power spectra, the rotation, the meridional flow, and the evolution rates all match fairly closely. While some of the rotation characteristics have been attributed to wave-like properties by Beck \& Schou (2000), Gizon et al. (2003), and Schou (2003), our simulated data are simply advected by a zonal flow (differential rotation) with speeds that never exceed those determined from helioseismology as reported by Howe et al. (2007).

While the similarities between the simulated data and the MDI data are strong, the differences are important and interesting. The rotation variations with depth and latitude were chosen to closely match those shown in Figure 1 of Howe et al. (2007). The differences in rotation rate seen at high latitudes in our Figure 4 suggest that the surface shear layer may not extend to the highest latitudes - a conclusion supported by the helioseismic measurements of Schou et al. (1998) and Corbard \& Thompson (2002). The differences in rotation rate seen at low wavenumbers in our Figure 5 suggest an increase in rotation across the surface shear layer of $1-2 \mathrm{nHz}$ near the equator.

The lack of excess power in the prograde components represented in Figure 6 for the simulation and the lack of evidence for a split between the prograde and retrograde components indicate differences in the evolution of the pattern. The manner in which we evolve the cells in the simulation is not fully satisfactory. The phase (and amplitude) changes associated with the evolving cells should replicate their advection by larger (non-axisymmetric) flows (Williams \& Cuntz 2009), their breakup into smaller cells, and their joining to form larger cells. The random changes in phase that we introduce to evolve the cells produce random offsets that are larger for larger wavenumbers and, when modulated by the power spectrum shown in Figure 3, produce the wedge of power shown in Figure 6 for the simulation. The split between prograde and retrograde movement seen in the MDI data suggests that the motions are not random but preferentially east-west. Lisle et al. (2004) found that supergranules appear to have a persistent north-south alignment and suggested that this was due to a north-south elongation of larger, giant cells that advect the supergranules to their boundaries. The rotational constraints on these giant cells (cf. Miesch et al. 2008, and references therein) should in fact give a preference for east-west flows near the equator and may produce an added preference for 
prograde flows. Note that this interpretation of the results does not involve wave-like properties for supergranules, but instead invokes simple advection by larger flows.

The advection of the supergranules by the meridional flow shows considerable promise. The fact that we fully and accurately recover the input velocity profile indicates that the latitudinal movement of supergranules provides new information on the meridional flow. The results with the MDI data indicate the presence of countercells (equatorward flow) at latitudes above about $65^{\circ}-70^{\circ}$. These results also indicate a meridional flow speed that matches that found for the small magnetic elements as measured by Komm et al. (1993) and Hathaway \& Rightmire (2010) but is significantly slower than that reported in the helioseismic determination of Giles et al. (1997) and Basu et al. (1999). The indication of a decrease in meridional flow speed with depth is also at odds with those helioseismic results but in agreement with the simulations of Hathaway (1982).

Magnetic element positions are known to coincide with the boundaries of supergranules and move as the supergranule boundaries evolve. This was shown fairly explicitly by Lisle et al. (2000). This suggests that the advection of the supergranules by the axisymmetric flows should be directly related to the advection of the magnetic elements. The differential rotation itself (relative to the Carrington rotation) has a velocity range from $\sim 40 \mathrm{~m} \mathrm{~s}^{-1}$ prograde to $\sim 180 \mathrm{~m} \mathrm{~s}^{-1}$ retrograde while the meridional flow has a peak velocity of only $\sim 11 \mathrm{~m} \mathrm{~s}^{-1}$. These weak flow velocities are no match for the flows of several 100 $\mathrm{m} \mathrm{s}^{-1}$ in the supergranules. The magnetic elements should be quickly carried to the boundaries and ultimately the interstices of the supergranules where the direct action of the differential rotation and meridional flow are too weak to dislodge them. The magnetic elements should experience differential rotation and meridional flow only to the extent that the supergranules themselves are advected by these flows at velocities representative of deeper layers.

The variation in the differential rotation and meridional flow with depth and latitude within the surface shear layer needs further examination. We have already argued for some changes from what was assumed in our simulation. In addition, we note that our association of different depths with different wavenumbers $\ell$ as indicated in Equations (9) and (13) suggests that cells with diameters $D=2 \pi R_{\odot} / \ell$ are advected by flows at depth $R_{\odot}-r \approx 0.82 D$. This would make typical supergranules extend to depths of $30 \mathrm{Mm}$. While this is deeper than suggested by the helioseismic investigations of Duvall et al. (1997) and Woodard (2007), those investigations were limited to layers not much deeper than $7 \mathrm{Mm}$. Shallower cells would suggest that the surface shear layer is shallower as well.

We thank NASA for its support of this research through a grant from the Heliophysics Guest Investigator Program to NASA Marshall Space Flight Center; $\mathrm{SOHO}$, which is a project of international cooperation between ESA and NASA; John Beck, who prepared the 31 minute filtered SOHO/MDI data used in this paper; and an anonymous referee whose comments led to significant improvements in both content and presentation.

\section{APPENDIX}

\section{SPECTRAL COEFFICIENT CHANGES DUE TO ADVECTION}

The changes in the spectral coefficients produced in the advection of the pattern by the axisymmetric flows are best illustrated using the radial component of the flow pattern as given by

$$
w(\theta, \phi, t)=\sum_{\ell=0}^{l \max } \sum_{m=-\ell}^{\ell} R_{\ell}^{m}(t) P_{\ell}^{m}(x) e^{i m \phi},
$$

where the time-varying spectral coefficient is $R_{\ell}^{m}(t), x=\cos \theta$, and $P_{\ell}^{m}$ is an Associated Legendre polynomial of angular degree $\ell$ and azimuthal order $m$. The Associated Legendre polynomials are normalized such that

$$
\int_{-1}^{1} P_{\ell}^{m}(x) P_{\ell^{\prime}}^{m^{\prime}}(x) d x=\delta_{\ell}^{\ell^{\prime}} \delta_{m}^{m^{\prime}} .
$$

The advection of this flow pattern by the axisymmetric flows is given by

$$
\frac{\partial w}{\partial t}=-\frac{V}{r} \frac{\partial w}{\partial \theta}-\frac{U}{r \sin \theta} \frac{\partial w}{\partial \phi} .
$$

The spherical harmonic representation gives explicit expressions for the spatial derivatives with

$$
\begin{aligned}
\frac{\partial w}{\partial \theta}= & \sum_{\ell=0}^{l \max } \sum_{m=-\ell}^{\ell} R_{\ell}^{m}(t) \frac{1}{\sin \theta} \\
& \times\left[\frac{\ell}{A_{\ell+1}^{m}} P_{\ell+1}^{m}(x)-\frac{(\ell+1)}{A_{\ell}^{m}} P_{\ell-1}^{m}(x)\right] e^{i m \phi}
\end{aligned}
$$

and

$$
\frac{\partial w}{\partial \phi}=\sum_{\ell=0}^{l \max } \sum_{m=-\ell}^{\ell} R_{\ell}^{m}(t) i m P_{\ell}^{m}(x) e^{i m \phi},
$$

where

$$
A_{\ell}^{m}=\left[\frac{(2 \ell+1)(2 \ell-1)}{(\ell+m)(\ell-m)}\right]^{\frac{1}{2}} .
$$

Taking the meridional velocity as

$$
V(\theta)=V_{0} \cos \theta \sin \theta
$$

and the azimuthal velocity as

$$
U(\theta)=r \sin \theta\left(\Omega_{0}+\Omega_{2} \cos ^{2} \theta+\Omega_{4} \cos ^{4} \theta\right),
$$

and then projecting Equation (A3) onto $Y_{\ell^{\prime}}^{m^{\prime}}$ gives

$$
\begin{aligned}
\frac{\partial R_{\ell^{\prime}}^{m^{\prime}}}{\partial t}= & -\frac{V_{0}}{r} \sum_{\ell=m^{\prime}}^{l \max } R_{\ell}^{m^{\prime}} \int_{-1}^{1}\left[\frac{\ell}{A_{\ell+1}^{m^{\prime}}} x P_{\ell+1}^{m^{\prime}}(x) P_{\ell^{\prime}}^{m^{\prime}}(x)\right. \\
& \left.-\frac{(\ell+1)}{A_{\ell}^{m^{\prime}}} x P_{\ell-1}^{m^{\prime}}(x) P_{\ell^{\prime}}^{m^{\prime}}(x)\right] d x \\
& -i m^{\prime} \Omega_{0} R_{\ell^{\prime}}^{m^{\prime}}-i m^{\prime} \Omega_{2} \sum_{\ell=m^{\prime}}^{l m a x} R_{\ell}^{m^{\prime}} \int_{-1}^{1} x^{2} P_{\ell}^{m^{\prime}}(x) P_{\ell^{\prime}}^{m^{\prime}}(x) d x \\
& -i m^{\prime} \Omega_{4} \sum_{\ell=m^{\prime}}^{l \max } R_{\ell}^{m^{\prime}} \int_{-1}^{1} x^{4} P_{\ell}^{m^{\prime}}(x) P_{\ell^{\prime}}^{m^{\prime}}(x) d x .
\end{aligned}
$$

The products of $x, x^{2}$, and $x^{4}$ with the Associated Legendre polynomials can be replaced with expressions containing only Associated Legendre polynomials of the same azimuthal order, $m$, using the recursion relation

$$
P_{\ell}^{m}(x)=A_{\ell}^{m} x P_{\ell-1}^{m}(x)-B_{\ell}^{m} P_{\ell-2}^{m}(x),
$$


where $A_{\ell}^{m}$ is given by Equation (A6) and

$$
B_{\ell}^{m}=\left[\frac{(2 \ell+1)(\ell+m-1)(\ell-m-1)}{(2 \ell-3)(\ell+m)(\ell-m)}\right]^{\frac{1}{2}} .
$$

Equation (A9) then reduces to (after dropping the primes)

$$
\begin{aligned}
& \frac{\partial R_{\ell}^{m}}{\partial t}=-\frac{V_{0}}{r}\left[\frac{(\ell-2)}{A_{\ell}^{m} A_{\ell-1}^{m}} R_{\ell-2}^{m}+\left[\frac{\ell B_{\ell+2}^{m}}{A_{\ell+2}^{m} A_{\ell+1}^{m}}-\frac{(\ell+1)}{A_{\ell}^{m} A_{\ell}^{m}}\right] R_{\ell}^{m}\right. \\
& \left.-\frac{(\ell+3) B_{\ell+2}^{m}}{A_{\ell+2}^{m} A_{\ell+2}^{m}} R_{\ell+2}^{m}\right]-i m \Omega_{0} R_{\ell}^{m}-i m \Omega_{2}\left[\frac{1}{A_{\ell}^{m} A_{\ell-1}^{m}} R_{\ell-2}^{m}\right. \\
& \left.+\left[\frac{B_{\ell+2}^{m}}{A_{\ell+2}^{m} A_{\ell+1}^{m}}+\frac{B_{\ell+1}^{m}}{A_{\ell+1}^{m} A_{\ell}^{m}}\right] R_{\ell}^{m}+\frac{B_{\ell+3}^{m}}{A_{\ell+3}^{m} A_{\ell+2}^{m}} B_{\ell+2}^{m} R_{\ell+2}^{m}\right] \\
& -i m \Omega_{4}\left[\frac{1}{A_{\ell}^{m} A_{\ell-1}^{m}} \frac{1}{A_{\ell-2}^{m} A_{\ell-3}^{m}}\right] R_{\ell-4}^{m}-i m \Omega_{4} \\
& \times\left[\frac{B_{\ell+2}^{m}}{A_{\ell+2}^{m} A_{\ell+1}^{m}}+\frac{B_{\ell+1}^{m}}{A_{\ell+1}^{m} A_{\ell}^{m}}+\frac{B_{\ell}^{m}}{A_{\ell}^{m} A_{\ell-1}^{m}}+\frac{B_{\ell-1}^{m}}{A_{\ell-1}^{m} A_{\ell-2}^{m}}\right] \frac{1}{A_{\ell}^{m} A_{\ell-1}^{m}} \\
& \times R_{\ell-2}^{m}-i m \Omega_{4}\left[\frac{B_{\ell+3}^{m}}{A_{\ell+3}^{m} A_{\ell+2}^{m}}+\frac{B_{\ell+2}^{m}}{A_{\ell+2}^{m} A_{\ell+1}^{m}}+\frac{B_{\ell+1}^{m}}{A_{\ell+1}^{m} A_{\ell}^{m}}\right] \\
& \times \frac{B_{\ell+2}^{m}}{A_{\ell+2}^{m} A_{\ell+1}^{m}} R_{\ell}^{m}-i m \Omega_{4}\left[\frac{B_{\ell+2}^{m}}{A_{\ell+2}^{m} A_{\ell+1}^{m}}+\frac{B_{\ell+1}^{m}}{A_{\ell+1}^{m} A_{\ell}^{m}}+\frac{B_{\ell}^{m}}{A_{\ell}^{m} A_{\ell-1}^{m}}\right] \\
& \times \frac{B_{\ell+1}^{m}}{A_{\ell+1}^{m} A_{\ell}^{m}} R_{\ell}^{m}-i m \Omega_{4}\left[\frac{B_{\ell+4}^{m}}{A_{\ell+4}^{m} A_{\ell+3}^{m}}+\frac{B_{\ell+3}^{m}}{A_{\ell+3}^{m} A_{\ell+2}^{m}}\right. \\
& \left.+\frac{B_{\ell+2}^{m}}{A_{\ell+2}^{m} A_{\ell+1}^{m}}+\frac{B_{\ell+1}^{m}}{A_{\ell+1}^{m} A_{\ell}^{m}}\right] \frac{B_{\ell+3}^{m}}{A_{\ell+3}^{m} A_{\ell+2}^{m}} B_{\ell+2}^{m} R_{\ell+2}^{m} \\
& -i m \Omega_{4}\left[\frac{B_{\ell+5}^{m}}{A_{\ell+5}^{m} A_{\ell+4}^{m}} \frac{B_{\ell+3}^{m}}{A_{\ell+3}^{m} A_{\ell+2}^{m}} B_{\ell+4}^{m} B_{\ell+2}^{m}\right] R_{\ell+4}^{m} \text {. }
\end{aligned}
$$

A common factor in Equation (A12) is of the form

$$
\frac{B_{\ell}^{m}}{A_{\ell}^{m} A_{\ell-1}^{m}}=\frac{(\ell+m-1)(\ell-m-1)}{(2 \ell-1)(2 \ell-3)} .
$$

The dominant terms on the right-hand side of Equation (A12) are those for the direct rotation of the $R_{\ell}^{m}$ coefficients. These terms are best handled analytically by taking

$$
R_{\ell}^{m}=\mathcal{R}_{\ell}^{m} e^{i m \Omega_{\ell}^{m} t},
$$

where

$$
\begin{aligned}
\Omega_{\ell}^{m}= & \Omega_{0}+\Omega_{2}\left[\frac{B_{\ell+2}^{m}}{A_{\ell+2}^{m} A_{\ell+1}^{m}}+\frac{B_{\ell+1}^{m}}{A_{\ell+1}^{m} A_{\ell}^{m}}\right] \\
& +\Omega_{4}\left[\frac{B_{\ell+3}^{m}}{A_{\ell+3}^{m} A_{\ell+2}^{m}}+\frac{B_{\ell+2}^{m}}{A_{\ell+2}^{m} A_{\ell+1}^{m}}+\frac{B_{\ell+1}^{m}}{A_{\ell+1}^{m} A_{\ell}^{m}}\right] \frac{B_{\ell+2}^{m}}{A_{\ell+2}^{m} A_{\ell+1}^{m}} \\
& +\Omega_{4}\left[\frac{B_{\ell+2}^{m}}{A_{\ell+2}^{m} A_{\ell+1}^{m}}+\frac{B_{\ell+1}^{m}}{A_{\ell+1}^{m} A_{\ell}^{m}}+\frac{B_{\ell}^{m}}{A_{\ell}^{m} A_{\ell-1}^{m}}\right] \frac{B_{\ell+1}^{m}}{A_{\ell+1}^{m} A_{\ell}^{m}} .
\end{aligned}
$$

With this substitution, Equation (A12) becomes an equation for the evolution of $\mathcal{R}_{\ell}^{m}$ in which the meridional flow (terms multiplied by $V_{0}$ ) couples the amplitude of the $\ell$ component to the $\ell \pm 2$ components while the differential rotation (terms multiplied by $\Omega_{2}$ and $\Omega_{4}$ ) couples the phase of the $\ell$ component to the $\ell \pm 2$ and $\ell \pm 4$ components. These spectral coefficients can be evolved in time using Equation (A12) with a fourth-order Runge-Kutta scheme. The solutions are stable and well behaved for quarter-hour time steps over at least 10 days for $\ell$ up to at least 1500 .

\section{REFERENCES}

Basu, S., Antia, H. M., \& Tripathy, S. C. 1999, ApJ, 512, 458

Beck, J. G., \& Giles, P. 2005, ApJ, 621, L153

Beck, J. G., \& Schou, J. 2000, Sol. Phys., 193, 333

Carrington, R. C. 1863, Observations of the Spots on the Sun from November 9, 1853, to March 24, 1861, Made at Redhill (London: Williams \& Norgate) Corbard, T., \& Thompson, M. J. 2002, Sol. Phys., 205, 211

Duvall, T. L., Jr. 1980, Sol. Phys., 66, 213

Duvall, T. L., et al. 1997, Sol. Phys., 170, 63

Foukal, P. 1979, ApJ, 218, 539

Foukal, P., \& Jokipii, R. 1975, ApJ, 199, L71

Giles, P. M., Duvall, T. L., Jr., Scherrer, P. H., \& Bogart, R. S. 1997, Nature, 390,52

Gilman, P. A., \& Foukal, P. 1979, ApJ, 229, 1179

Gizon, L., Duvall, T. L., Jr., \& Schou, J. 2003, Nature, 421, 43

Hart, A. B. 1954, MNRAS, 114, 17

Hathaway, D. H. 1982, Sol. Phys., 77, 341

Hathaway, D. H. 1987, Sol. Phys., 108,

Hathaway, D. H. 1988, Sol. Phys., 117, 329

Hathaway, D. H. 1992, Sol. Phys., 137, 15

Hathaway, D. H., Beck, J. G., Bogart, R. S., Bachmann, K. T., Khatri, G., Petitto, J. M., Han, S., \& Raymond, J. 2000, Sol. Phys., 193, 299

Hathaway, D. H., Beck, J. G., Han, S., \& Raymond, J. 2002, Sol. Phys., 205, 25

Hathaway, D. H., \& Rightmire, L. 2010, Science, 327, 1350

Hathaway, D. H., Williams, P. E., \& Cuntz, M. 2006, ApJ, 644, 598

Howard, R., \& Harvey, J. 1970, Sol. Phys., 12, 23

Howe, R., Christensen-Dalsgaard, J., Hill, F., Komm, R., Schou, J., Thompson, M. J., \& Toomre, J. 2007, Adv. Space Res., 40, 915

Komm, R. W., Howard, R. F., \& Harvey, J. W. 1993, Sol. Phys., 147, 207

Korzennik, S. G., Rebello-Soares, M. C., \& Schou, J. 2004, ApJ, 602, 481

Leighton, R. B. 1964, ApJ, 140, 1559

Leighton, R. B., Noyes, R. W., \& Simon, G. W. 1962, ApJ, 135, 474

Lisle, J., De Rosa, M., \& Toomre, J. 2000, Sol. Phys., 197, 21

Lisle, J. P., Rast, M. P., \& Toomre, J. 2004, ApJ, 608, 1167

Meunier, N., \& Roudier, T. 2007, A\&A, 466, 691

Meunier, N., Tkaczak, R., \& Roudier, T. 2007, A\&A, 463, 745

Miesch, M. S., Brun, A. S., De Rosa, M. L., \& Toomre, J. 2008, ApJ, 673, 557

Mosher, J. M. 1977, PhD dissertation, California Institute of Technology

November, L. J., Toomre, J., Gebbie, K. B., \& Simon, G. W. 1981, ApJ, 245 L123

Rieutord, M., \& Rincon, F. 2010, Living Rev. Sol. Phys., 7, 2

Scherrer, P. H., et al. 1995, Sol. Phys., 162, 129

Schou, J. 2003, ApJ, 596, L259

Schou, J., et al. 1998, ApJ, 505, 390

Schrijver, C. J., \& Martin, S. F. 1990, Sol. Phys., 129, 95

Simon, G. W., \& Leighton, R. B. 1964, ApJ, 140, 1120

Simon, G. W., Title, A. M., \& Weiss, N. O. 1995, ApJ, 442, 886

Snodgrass, H. B., \& Ulrich, R. K. 1990, ApJ, 351, 309

Thompson, M. J., et al. 1996, Science, 272, 1300

van Ballegooijen, A. A., Cartledge, N. P., \& Priest, E. R. 1998, ApJ, 501, 866

Wang, Y.-M., Sheeley, N. R., Jr., \& Lean, J. 2002, ApJ, 580, 1188

Williams, P. E., \& Cuntz, M. 2009, A\&A, 505, 1265

Woodard, M. F. 2007, ApJ, 668, 1189

Worden, S. P. 1975, Sol. Phys., 45, 521 